\title{
Laparoscopic surgery in a patient with atypical presentation of COVID-19: salient points to reduce the perils of surgery
}

Singapore Med J 2020; 61(8): 443-444 https://doi.org/10.11622/smedj.2020086

Dear Sir,

As the coronavirus disease 2019 (COVID-19) pandemic continues to leave indelible marks on all aspects of society, hospitals at the front line have shifted their resources and restructured existing protocols and practices to handle this crisis on an unprecedented scale. The most common symptoms of COVID-19 are fever and cough. (1) However, it is pivotal to be aware of atypical abdominal manifestations of COVID-19 or patients with surgical conditions who concurrently harbour asymptomatic COVID-19 infection, as we illustrate from our recent experience. In this case, we highlight some surgical conundrums we faced in the management of an acute surgical patient who presented with undetected COVID-19.

A 66-year-old man with minimal comorbidities presented with two days of right hypochondrium pain and fever. Clinical examination revealed a temperature of $38.3^{\circ} \mathrm{C}$, blood pressure of $125 / 75 \mathrm{mmHg}$ and a saturation of $96 \%$ on room air. His right hypochondrium was tender on palpation and Murphy's sign was positive. Total white blood cell count was normal at $6.38 \times 10^{9} / \mathrm{L}$ (normal range 3.82-9.91 × 10\% /L) with associated lymphopenia of $1.04 \times 10^{9} / \mathrm{L}$ (normal range $1.13-3.49 \times 10^{9} / \mathrm{L}$ ). Computed tomography showed the presence of a large gallstone with stranding at the gallbladder fundus. The patient did not have any respiratory symptoms nor any recent travel history or infective contacts suspicious of COVID-19. His chest radiograph was also unremarkable on admission.

Routine laparoscopic cholecystectomy was performed based on a presumptive diagnosis of acute cholecystitis. The surgery was conducted uneventfully with intraoperative findings of an oedematous gallbladder with mild inflammation and the presence of a large gallstone (Fig. 1). Histology eventually showed chronic cholecystitis. Postoperatively, the patient continued to have persistent fever spikes and eventually developed acute respiratory failure on Postoperative Day 3, requiring prompt intubation and subsequent care in the intensive care unit (ICU). Chest radiography findings showed progressive bilateral pneumonitis (Fig. 2) and a subsequent swab was positive for COVID-19.

As this was the first case of laparoscopic surgery for symptomatic gallstone disease performed on a COVID-19 patient in Singapore, although it was unexpected, we herein share a few key lessons that we hope may benefit the wider surgical community.

First, patients with acute surgical pathology may have a concurrent asymptomatic COVID-19 infection that remains undetected until after the patient has undergone surgery. Lei et $\mathrm{al}^{(2)}$ have reported increased rates of mortality of up to $20.5 \%$ along with increased postoperative morbidity and ICU admissions when patients with COVID-19 are subjected to surgical stress. Although index admission laparoscopic cholecystectomy for patients with acute biliary symptoms is widely considered the standard approach for management of such patients to avoid re-admissions and life-threatening complications, a more cautious approach should be heeded during this pandemic. The latest American College of Surgeons guidelines and the harmonised United Kingdom guidelines also recommend treating acute biliary disease conservatively when possible to avoid potential increased morbidity in the surgical patient with concurrent COVID-19. (3,4) $^{(3)}$

Second, a robust screening protocol to detect COVID-19 is essential to guard the safety of patients and healthcare workers. We were caught unawares by our case of acute cholecystitis and concurrent asymptomatic COVID-19 infection. The American Society of Anesthesiologists and Anesthesia Patient Safety Foundation joint statement on perioperative testing for COVID-19 recommended that

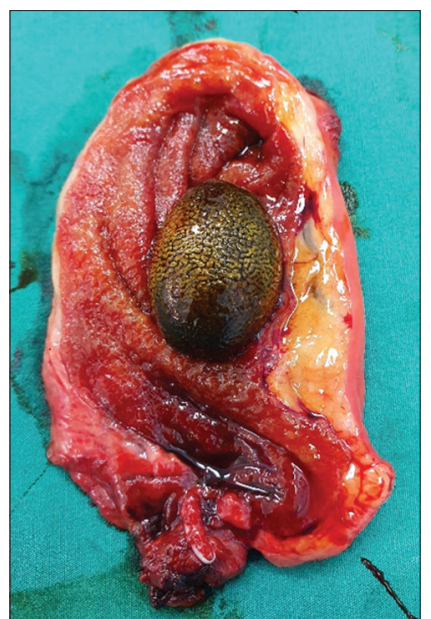

Fig. 1 Photograph shows an oedematous gallbladder with mild inflammation and the presence of a large gallstone.

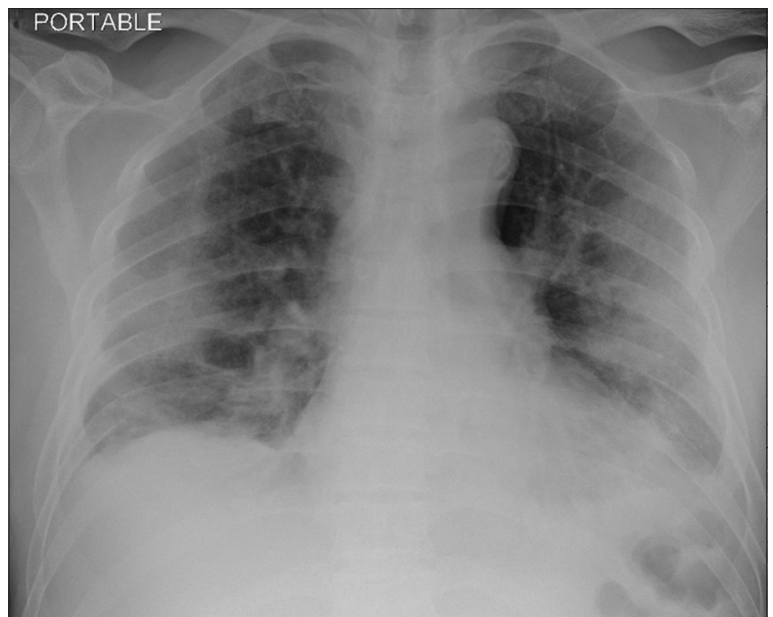

Fig. 2 Radiograph shows bilateral lung infiltrates indicative of pneumonia on Postoperative Day 2. 
all patients undergoing non-emergent surgery should undergo nucleic acid amplification testing (including polymerase chain reaction tests) prior to surgery, where infections are prevalent. ${ }^{(5)}$ If the test is negative, the surgeon can proceed with standard precautions. If the test is positive, less invasive options may be considered such as percutaneous drainage and prolonged antibiotic treatment. If surgery is still deemed necessary, the patient has to be counselled appropriately regarding the potential morbidity of worsening respiratory compromise and prolonged ICU stay, as demonstrated in this case. A risk-stratified COVID-19 screening approach is similarly needed to identify patients with surgical emergencies who harbour asymptomatic infection.

Third, the risk of COVID-19 transmission during laparoscopic surgery is still largely unknown despite some research showing that laparoscopy can lead to aerosolisation of blood-borne viruses. ${ }^{(6)}$ The decision on whether to perform laparoscopic or open surgery is still debatable; however, a review conducted by Chadi et $\mathrm{al}^{(7)}$ suggested that laparoscopic surgery poses significant advantages in mitigating inherent risks of transmission, as it creates a more regulated closed environment with controlled inflow and outflow of air. The Society of American Gastrointestinal and Endoscopic Surgeons and the European Association for Endoscopic Surgery recently devised specific protocols for the conduct of laparoscopic surgery, recommending the use of a smoke evacuation system or filtration device. ${ }^{(8)}$ Although such devices were not employed in our case, it is fortunate that the three operating surgeons who were wearing standard surgical masks did not contract COVID-19. However, it is imperative that strict use of personal protective equipment (PPE) must be considered when performing surgery (be it open or laparoscopic) with, at a minimum, N95 masks and face shields, to ensure that the safety of the entire team is not compromised. Our institutional PPE donning protocol in the operating theatre was subsequently modified as a result of this case.

The above lessons gleaned from this experience reinforce the need for surgeons to have heightened awareness of atypical presentations of COVID-19 patients that may masquerade as acute surgical conditions and to have clear protocols for PPE donning during surgery. A thoughtful and concerted effort within each department is also necessary to develop robust frameworks to ensure safe provision of surgery during this pandemic.

Yours sincerely,

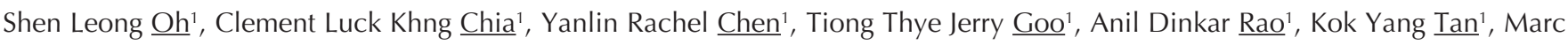
Weijie Ong $^{1}$

${ }^{1}$ Department of General Surgery, Khoo Teck Puat Hospital, Singapore. ong.marc.wj@ktph.com.sg

\section{References}

1. Wang D, Hu B, Hu C, et al. Clinical characteristics of 138 hospitalized patients with 2019 novel coronavirus-infected pneumonia in Wuhan, China. JAMA 2020; 323:1061-9

2. Lei S, Jiang F, Su W, et al. Clinical characteristics and outcomes of patients undergoing surgeries during the incubation period of COVID-19 infection. EClinicalMedicine 2020 Apr 5. https://doi.org/10.1016/j.eclinm.2020.100331. [Epub ahead of print]

3. The Association of Coloproctology of Great Britain and Ireland. Urgent Intercollegiate General Surgery Guidance on COVID-19. 25 March 2020. Available at: https:// www.acpgbi.org.uk/news/urgent-intercollegiate-general-surgery-guidance-on-covid-19/. Accessed April 4, 2020.

4. American College of Surgeons. COVID-19: Elective Case Triage Guidelines for Surgical Care. 24 March 2020. Available at: https://www.facs.org/covid-19/clinicalguidance/elective-case/emergency-surgery. Accessed April 4, 2020.

5. American Society of Anesthesiologists. The ASA and APSF Joint Statement on Perioperative Testing for the COVID-19 Virus. 29 April 2020. Available at: https://www. asahq.org/about-asa/newsroom/news-releases/2020/04/asa-and-apsf-joint-statement-on-perioperative-testing-for-the-covid-19-virus. Accessed May 1, 2020.

6. Englehardt RK, Nowak BM, Seger MV, Duperier FD. Contamination resulting from aerosolized fluid during laparoscopic surgery. JSLS 2014; 18 :e2014.00361.

7. Chadi SA, Guidolin K, Caycedo-Marulanda A, et al. Current evidence for minimally invasive surgery during the COVID-19 pandemic and risk mitigation strategies: a narrative review. Ann Surg 2020. Available at: https://journals.Iww.com/annalsofsurgery/Documents/Current\%20Evidence\%20for\%20Minimally\%20Invasive\%20 Surgery\%20.pdf. Accessed May 5, 2020.

8. Society of American Gastrointestinal and Endoscopic Surgeons. SAGES and EAES recommendations regarding surgical response to COVID-19 crisis. Available at: 29 March 2020. Available at: https://www.sages.org/recommendations-surgical-response-covid-19/. Accessed April 2, 2020. 